\title{
BMJ open Does the effect of vitamin A supplements depend on vaccination status? An observational study from Guinea-Bissau
}

\author{
Ane B Fisker, ${ }^{1,2,3}$ Peter Aaby, ${ }^{1,2}$ Carlito Bale, ${ }^{1}$ Ibraima Balde, ${ }^{1}$ \\ Sofie Biering-Sørensen, ${ }^{1,2}$ Jane Agergaard, ${ }^{1,4}$ Cesario Martins, ${ }^{1}$ \\ Bo M Bibby, ${ }^{3}$ Christine S Benn ${ }^{1,2}$
}

To cite: Fisker $A B$, Aaby $P$, Bale $C$, et al. Does the effect of vitamin A supplements depend on vaccination status? An observational study from Guinea-Bissau. BMJ Open 2012;2:e000448. doi:10.1136/

bmjopen-2011-000448

- Prepublication history and additional figure for this paper are available online. To view these files please visit the journal online (http:// bmjopen.bmj.com).

Received 15 October 2011 Accepted 19 December 2011

This final article is available for use under the terms of the Creative Commons Attribution Non-Commercial 2.0 Licence; see http://bmjopen.bmj.com

${ }^{1}$ Bandim Health Project, INDEPTH Network, Bissau, Guinea-Bissau

${ }^{2}$ Bandim Health Project, Statens Serum Institut, Copenhagen S, Denmark ${ }^{3}$ Department of Biostatistics, Institute of Public Health, University of Aarhus, Aarhus, Denmark

${ }^{4}$ Department of Infectious Diseases, Aarhus University Hospital, Skejby Sygehus,

Aarhus, Denmark

Correspondence to

Dr Ane B Fisker;

a.fisker@bandim.org

\section{ABSTRACT}

Objective: Vitamin A supplementation (VAS) is estimated to reduce all-cause mortality by $24 \%$. Previous studies indicate that the effect of VAS may vary with vaccination status. The authors evaluated the effect of VAS provided in campaigns on child survival overall and by sex and vaccination status at the time of supplementation.

Design: Observational cohort study.

Setting and participants: The study was

conducted in the urban study area of the Bandim Health Project in Guinea-Bissau. The authors documented participation or non-participation in two national vitamin A campaigns in December 2007 and July 2008 for children between 6 and 35 months of age. Vaccination status was ascertained by inspection of vaccination cards. All children were followed prospectively.

Outcome measures: Mortality rates for supplemented and non-supplemented children were compared in Cox models providing mortality rate ratios (MRRs).

Results: The authors obtained information from $93 \%$ of 5567 children in 2007 and $90 \%$ of 5799 children in 2008. The VAS coverage was $58 \%$ in 2007 and $68 \%$ in 2008. Mortality in the supplemented group was $1.5 \%$ (44 deaths/2873 person-years) and $1.6 \%$ (20 deaths/1260 person-years) in the non-supplemented group (adjusted MRR $=0.78$ $(0.46 ; 1.34))$. The effect was similar in boys and girls. Vaccination cards were seen for $86 \%$ in 2007 and $84 \%$ in 2008. The effect of VAS in children who had measles vaccine as their last vaccine (2814 children, adjusted $M R R=0.34(0.14 ; 0.85))$ differed from the effect in children who had diphtheria-tetanus-pertussis vaccine as their last vaccine (3680 children, adjusted MRR=1.29 (0.52; 3.22), $p=0.04$ for interaction).

Conclusion: The effect of VAS differed by most recent vaccination, being beneficial after measles vaccine but not after diphtheria-tetanus-pertussis vaccine.

\section{ARTICLE SUMMARY}

Article focus

- Vitamin A supplementation (VAS) is estimated to reduce all-cause mortality by $24 \%$.

- The effect of VAS may vary with vaccination status, being beneficial with or after measles vaccine (MV) but not after diphtheriatetanus-pertussis (DTP) vaccine.

Key messages

- The effect of VAS is heterogeneous.

- The effect of VAS varied with vaccination status: supplemented children had lower mortality than non-supplemented children when MV was the most recent vaccine but not when DTP was the most recent vaccine.

- The effect of VAS tended to differ by season of supplementation.

Strengths and limitations of this study

- Information was collected on the individual level, and the children were followed prospectively.

- Due to the observational nature of the study, the comparison of supplemented and non-supplemented children should be interpreted with caution.

- However, a selection bias is unlikely to have worked in different directions for children who had DTP and MV as the most recent vaccine.

\section{INTRODUCTION}

Vitamin A supplementation (VAS) is estimated to reduce all-cause mortality by $24 \%$ when provided to children aged 6-59 months at four to six monthly intervals. ${ }^{1}$ However, according to a preliminary report ${ }^{2}$ of a recent cluster randomised trial involving $>1$ million Indian children, VAS compared with no VAS was associated with no survival benefit. We have hypothesised that the effect of VAS varies with vaccination status because VAS amplifies the non-specific 
immunological effects of vaccines. ${ }^{3}$ Hence, VAS is beneficial in the time window when BCG (recommended at birth) and measles vaccine (MV, recommended at 9 months of age) are the most recent vaccines but not while diphtheria-tetanus-pertussis vaccine (DTP, recommended at 6,10 and 14 weeks of age) is the most recent vaccine. ${ }^{3}$ The hypothesis provides an explanation of the lack of a beneficial effect of VAS in children aged 1-5 months, ${ }^{45}$ the age group in which DTP is the predominant vaccine. The hypothesis also offers an explanation of why the large mortality reduction reported by the meta-analysis of the trials between 1986 and $1993^{1}$ may no longer be reproducible; these trials were conducted before the Expanded Programme on Immunisations reached high coverage. ${ }^{6}$ We recently reanalysed data from the 1989 to 1991 VAS trial in Ghana, which had reported an overall mortality reduction of $19 \%(2 \% ; 32 \%) .{ }^{7}$ The reanalysis revealed that the survival advantage was limited to children with no health card (a proxy for being unvaccinated). Among vaccinated children, there was a harmful effect of VAS among girls likely to receive DTP during follow-up. ${ }^{8}$

WHO recommends vitamin A for all children aged 6-59 months distributed in campaigns or at vaccination contacts in areas where vitamin A deficiency is a public health problem. ${ }^{9}{ }^{10}$ In Guinea-Bissau, VAS is distributed in biannual campaigns, often linked to other interventions such as distribution of mebendazole, oral polio vaccine (OPV) or other vaccines. ${ }^{11}$ Two different strategies have been employed in the urban area: fixed post campaigns where the mother brings her child to the campaign team and door-to-door campaigns where all houses are visited by the campaign team. The fixed post campaigns require less staff but have lower coverage. In December 2007 and June/July 2008, fixed post campaigns distributing vitamin $\mathrm{A}$ and mebendazole were conducted. We registered all children receiving VAS and mebendazole during these campaigns and assessed survival prospectively to compare survival in supplemented and non-supplemented children overall and by sex and vaccination status.

\section{SUBJECTS AND METHODS \\ Setting}

The Bandim Health Project (BHP) operates a health and demographic surveillance system (HDSS) covering a population of 102000 people in six suburban districts of Bissau, the capital of Guinea-Bissau, West Africa. All households are visited monthly to register new births, pregnancies and deaths. Children below the age of 3 are followed through three monthly visits to ascertain vital status and vaccination status. At the first visit, after birth information on socioeconomic status is collected. The indicators include maternal characteristics (education and ethnicity) and household characteristics (type of roofing, availability of bathroom and electricity). All children aged 6-35 months living in the urban study area on the first day of the VAS campaigns in 2007 and
2008 were eligible for the present study. Since children below 3 years of age are followed intensively through the HDSS, these children have been the focus for the present study.

WHO classifies Guinea-Bissau as having a public health problem of VAS. ${ }^{12}$ We have previously found that $16 \%$ of 4-month-old children were vitamin A deficient (retinolbinding protein concentration equivalent to plasma retinol $<0.70 \mu \mathrm{mol} / \mathrm{l}) .{ }^{13}$ During $2007-2008$, we assessed vitamin A status in 181 children aged 6-17 months presenting for vaccinations and found that $70 \%$ of the children had a retinol-binding protein concentration equivalent to plasma retinol $<0.70 \mu \mathrm{M}$ (unpublished data).

\section{Campaign information}

National VAS campaigns were conducted by the Ministry of Health from 14 to 18 December 2007 and 30 June to 4 July 2008. Children aged 6-11 months received $100000 \mathrm{IU}$ vitamin A, children aged 12-35 months received $200000 \mathrm{IU}$ vitamin A and $250 \mathrm{mg}$ mebendazole and children aged 36-59 months received $200000 \mathrm{IU}$ vitamin A and $500 \mathrm{mg}$ mebendazole. Trained BHP field assistants were present at all posts. The field assistants brought a list of all children registered in the area and noted on this list whether a child had received VAS and mebendazole at the post. The lists contained information on vaccines registered prior to the campaign. If the vaccination card was seen, the assistant noted this on the list, verified the already registered vaccines and updated the information if new vaccines had been received.

During the weeks after the campaigns, all children who had not been seen during the campaigns were visited. The caretaker was asked whether the child had received VAS elsewhere. The vaccination card was seen and information on vaccines verified and updated. If the child and caretaker were not present, the household was visited up to three times, and if no one could provide information, the campaign status was classified as unknown.

During the 2007 campaign, we discovered that the vitamin A capsules given in two of the six districts had been produced in November 2004 and had passed the expiry date 1 month before. The capsules were immediately replaced by a new batch. We sent two of the expired 200000 IU capsules to 'as Vitas Oslo Innovation Center' (Oslo, Norway) to have their vitamin A content measured. The vitamin A contents were slightly lower than expected: $167569 \mathrm{IU}$ and $154863 \mathrm{IU}$. Capsules used in the 2008 campaign were all from a new stock.

\section{Vaccine information}

The vaccination schedule in Guinea-Bissau when this study was conducted was BCG and OPV at birth, three doses of DTP vaccines at 6,10 and 14 weeks of age and MV at 9 months of age. A booster DTP vaccine at 18 months of age was given in the routine vaccination programme until March 2007 after which it was only 
given in a randomised trial (trial A below). In August 2008, the DTP vaccine at 6,10 and 14 weeks was replaced by the pentavalent (DTP-HiB-HBV) vaccine and a yellow fever vaccine was added to be given with MV at 9 months of age.

During the study period, three trials of alternative vaccination strategies took place in the BHP area. First, between October 2005 and October 2009, trial A enrolled children aged 18 months in a randomised trial of booster DTP. Children who had completed their primary immunisation schedule of three doses of DTP and an MV were randomised to receive booster DTP $+\mathrm{OPV}$ or OPV only. Second, between August 2003 and April 2007, trial B enrolled children aged 4.5 months in a randomised trial of early MV. Children were randomised to an extra dose of MV at 4.5 months or no vaccine; a subgroup of the controls received an extra MV at 18 months of age. ${ }^{14}$ Third, between October 2005 and April 2008, trial C enrolled children aged at least 9 months who were delayed in relation to the vaccination schedule and presented at the health centres to receive MV and the third DTP vaccine. They were randomised to receive both vaccines and booster DTP at 18 months of age versus MV only and no booster DTP ${ }^{15}$ (supplementary figure). Furthermore, in May 2006, a measles vaccination campaign was conducted in which children aged 6 months to 14 years were given an MV; thus, the oldest children in the present study often had MV received in this campaign as their most recent vaccine.

For the present study, vaccine status was ascertained during the campaigns. Furthermore, as part of the BHP routines, vaccines are registered daily at the three health centres in the study area and through the tri-monthly home visits and as a part of the trials described above. We linked the campaign information with information from the above sources, using only information collected prior to the campaign. In case of discrepancy, information from the trials was considered superior. If no trial information was available and if two of the other sources agreed, this information was accepted. Vaccination information obtained during the campaign was updated for 12\% (1088/8812) of the children: $9 \%$ based on data collected at the health centres, $1 \%$ based on routine surveillance data and $2 \%$ based on trial data. Except for children included in trial A, OPV is almost always given with BCG or DTP. In line with previous studies, ${ }^{11} 16$ children who received OPV along with another vaccine were classified according to the co-administered vaccine, that is, children who received DTP alone (161 children) and DTP+OPV (3519 children) were classified as DTP and children who received MV+OPV (205 children) and MV alone (2609) were classified as MV.

\section{Follow-up}

All children below 3 years of age were followed by the HDSS. For children who died, a locally adapted version of the INDEPTH verbal autopsy ${ }^{17}$ was conducted. Cause of death was determined by a local paediatrician.

\section{Statistical analyses}

Children entered the analysis on the day we knew their VAS campaign status (supplemented, non-supplemented or no information). Thus, a child who was registered to have participated in the campaign during the days of the campaign contributed time at risk from the day of supplementation. A child who had campaign status registered after the campaign contributed time from the day the information was obtained. Follow-up of children was censored when a subsequent campaign was initiated (30 June 2008 for the 2007 campaign and 9 January 2009 for the 2008 campaign), 3 years of age, migration or reception of VAS in a trial, whichever came first. Some children were enrolled in a randomised trial of VAS at vaccination contacts after 6 months of age (Trial registration: clinicaltrials.gov, NCT00514891). These children were censored from follow-up on the date of receiving VAS in the trial (figure 1).

We examined whether the background factors were evenly distributed between children in the VAS, no VAS and no information group, as well as between the two main groups (VAS and no VAS) using $\chi^{2}$ tests for categorical variables and rank-sum test for age at the time of the campaign.

Survival was examined in Cox proportional hazards models with age as underlying time; thus, age was inherently controlled for in all models. The proportional hazards assumption was tested using Schoenfeld residuals. Crude and adjusted estimates are presented. The 2007 campaign took place in the dry season and the 2008 campaign in the rainy season. A priori we had decided to adjust all estimates for sex and campaign since sex and season of supplementation have been shown to be important determinants of the effect of VAS in our previous studies. ${ }^{8} 1819$ We furthermore adjusted the comparison of supplemented and non-supplemented children for the background factors which were associated with participation in the VAS campaigns. In all analyses, we investigated whether the survival of supplemented and non-supplemented children varied by sex and campaign (season). We furthermore investigated interactions with vaccination status. Interaction between VAS and potential effect modifier was evaluated by Wald statistics. Among children who had OPV as the most recent vaccine prior to the campaign, no deaths occurred among children who did not receive VAS. Therefore, the potential interaction between VAS and OPV was tested by Mantel-Haenszel stratification. Finally, previous studies have indicated that the effect of VAS in girls depends on whether or not they have received VAS on a previous occasion. ${ }^{20}{ }^{21}$ We therefore investigated whether survival after the 2008 campaign varied with reception of VAS in the 2007 campaign.

\section{RESULTS}

In the 2007 campaign, we followed 5567 (92\%) of the 6026 children aged $6-35$ months registered in the study area. Reasons for losses to follow-up are given in figure 1 . 
Figure 1 Flowchart of children eligible for campaign participation. BHP, Bandim Health Project; RCT, randomised controlled trial; VAS, vitamin A supplementation.

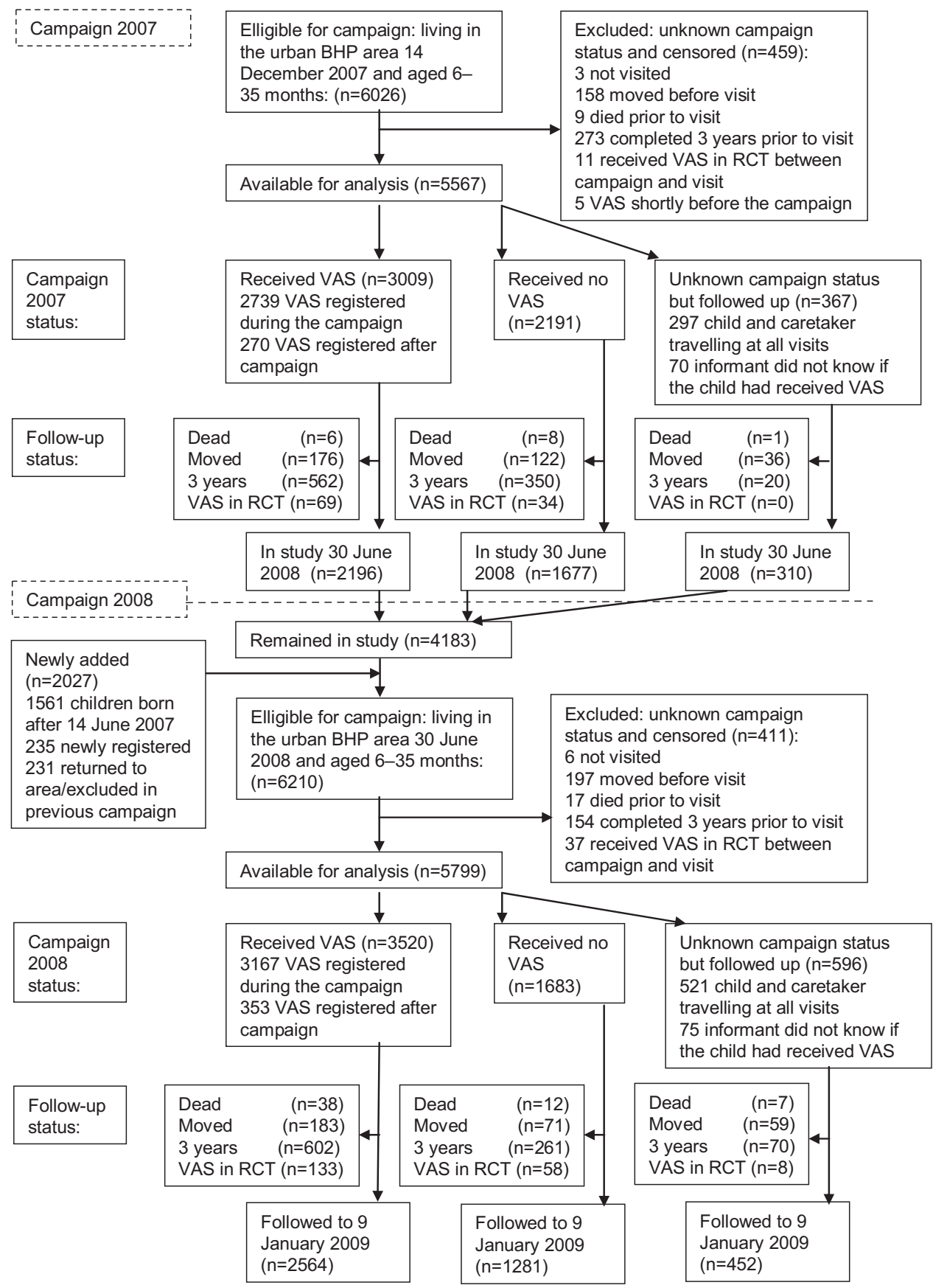

For $7 \%(367 / 5567)$, we obtained no information on campaign status since the family was travelling at all visits. Among the remaining 5200 children, 3009 (58\%) had received VAS and 2191 (42\%) had not. In the 2008 campaign, we followed 5799 (93\%) of 6210 children. For $10 \%(596 / 5799)$, we were unable to obtain information on campaign status. Among the remaining 5203 children, 3520 (68\%) had received VAS and 1683 (32\%) had not (figure 1). Median length of follow-up and IQR was 6.2 (4.4-6.4) months for supplemented, 4.2 (3.2-5.1) months for non-supplemented and 2.9 (1.8-3.4) months for children for whom we did not obtain information.

The distribution of background factors is shown in table 1. Children for whom we had no information on campaign status had lower socioeconomic status, were more likely to be from the Muslim ethnic groups (Fula and Mandinga) and were younger. These children also tended to have higher mortality, crude mortality rate ratio $(\mathrm{MRR})=2.17(0.96 ; 4.94) \quad($ table 2$)$. In contrast, there were only few differences in background factors between supplemented and non-supplemented children. Non-supplemented children were more likely to have mothers with no formal education $(p<0.001)$, to belong to the Muslim ethnic groups $(p<0.001)$ and were less likely to have their vaccination card inspected $(p<0.001)$. Furthermore, more supplemented children were enrolled in trials and as a result more supplemented children had received OPV as the most recent vaccine in $2007(p=0.05)$. In 2008, the distribution of 


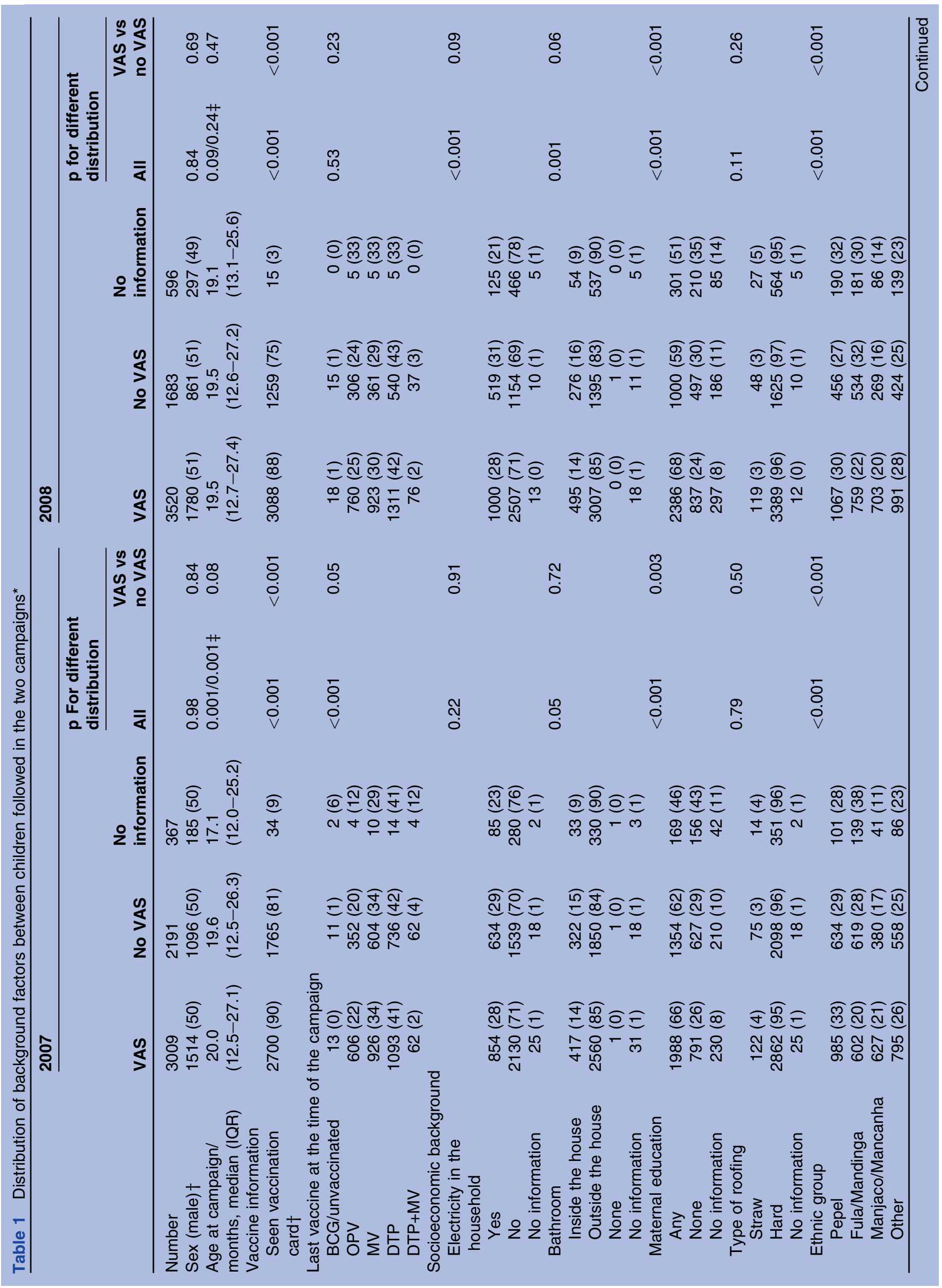




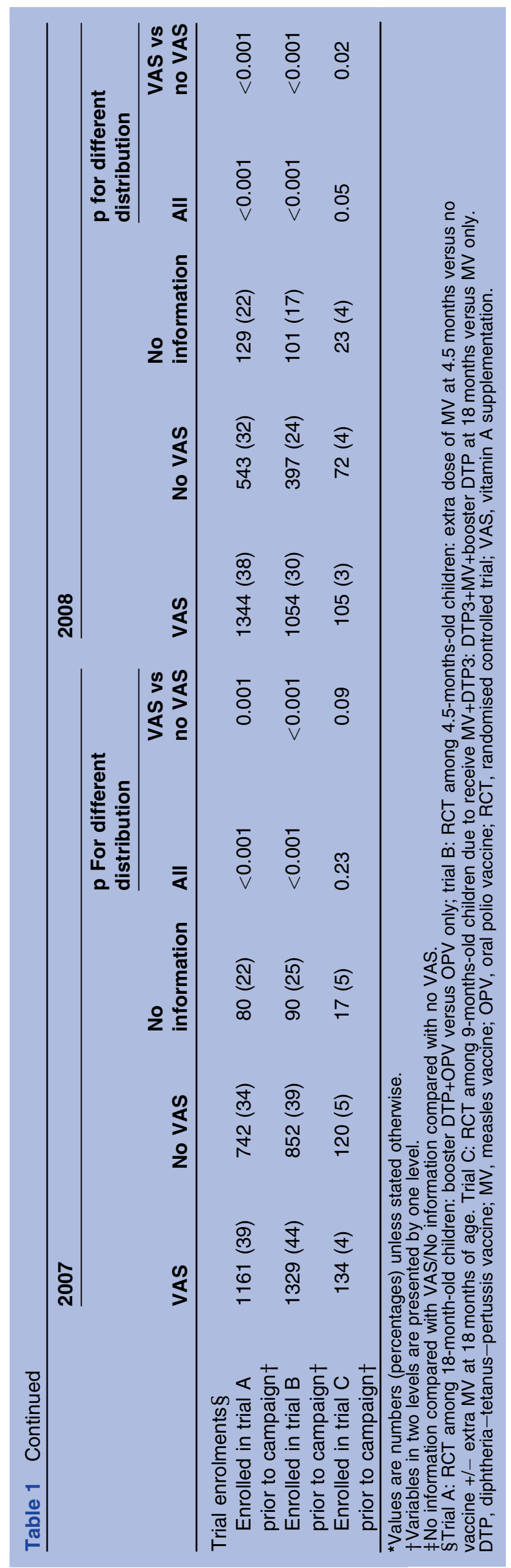

different vaccines did not differ between supplemented and non-supplemented children $(\mathrm{p}=0.23)$ (table 1).

\section{Effect of VAS}

There was no significant difference in mortality between supplemented and non-supplemented children, the crude MRR being $0.93(0.55 ; 1.58)$. When the estimate was adjusted for sex, campaign, maternal education, ethnicity and inspected vaccination card, the difference in mortality between supplemented and non-supplemented children was $0.78(0.46 ; 1.34)$ (table 2). The effect did not differ significantly for children aged 6-11 months who received $100000 \mathrm{IU}$ vitamin A (adjusted $\mathrm{MRR}=1.14(0.41 ; 3.19))$ and children above 12 months who received $200000 \mathrm{IU}$ vitamin $\mathrm{A}$ and $250 \mathrm{mg}$ mebendazole (adjusted MRR $=0.69(0.36 ; 1.29)$ ) $(p=0.41$, test of interaction) (table 2$)$. The effect did not differ for boys and girls $(p=0.72)$.

Mortality was much higher in the rainy season after the 2008 campaign (57 deaths/2208 person-years) than in the dry season after the 2007 campaign ( 15 deaths/2154 person-years); the adjusted MRR was 3.79 (2.13; 6.74) comparing 2008 versus 2007. The difference between supplemented and non-supplemented tended to differ between the two campaigns; the adjusted MRR was 0.38 $(0.13 ; 1.10)$ after the 2007 campaign and $1.02(0.53$; 1.96) after the 2008 campaign $(\mathrm{p}=0.12$, test of interaction). This difference may have been strongest for girls; the adjusted MRR was $0.16(0.02 ; 1.59)$ after the 2007 campaign and $1.32(0.44 ; 3.94)$ after the 2008 campaign $(\mathrm{p}=0.11$, test of interaction), whereas there was no evidence of a difference in boys $(p=0.50)$ (table 2). Further adjusting for last vaccine at the time of the campaign did not change the conclusions.

The difference between supplemented and nonsupplemented children varied with maternal education. Supplementation was associated with lower mortality in children of educated mothers (adjusted $\mathrm{MRR}=0.41$ $(0.21 ; 0.80))$ but not in children of mothers with no schooling (adjusted $\mathrm{MRR}=1.78(0.60 ; 5.31)) \quad(\mathrm{p}=0.02$, test of interaction). The effect did not vary with the other socioeconomic indicators presented in table 1 (data not shown).

\section{Effect of VAS by vaccination status}

Vaccination cards were seen for $86 \%$ of the children in 2007 and $84 \%$ in 2008, with more cards seen for supplemented compared with non-supplemented children (2007: $90 \%$ vs $81 \%, \mathrm{p}<0.001$; $2008: 88 \%$ vs $75 \%$, $\mathrm{p}<0.001$, table 1$) ; 9 \%(6 / 64)$ of the deaths in the study were excluded due to no information on vaccination status.

The difference in mortality between supplemented and non-supplemented children varied with vaccination status. In the 2814 children who had received MV as the most recent vaccine prior to the campaign, the adjusted MRR was $0.34(0.14 ; 0.85)$. In the 3680 children who had received DTP as the most recent vaccine, the 


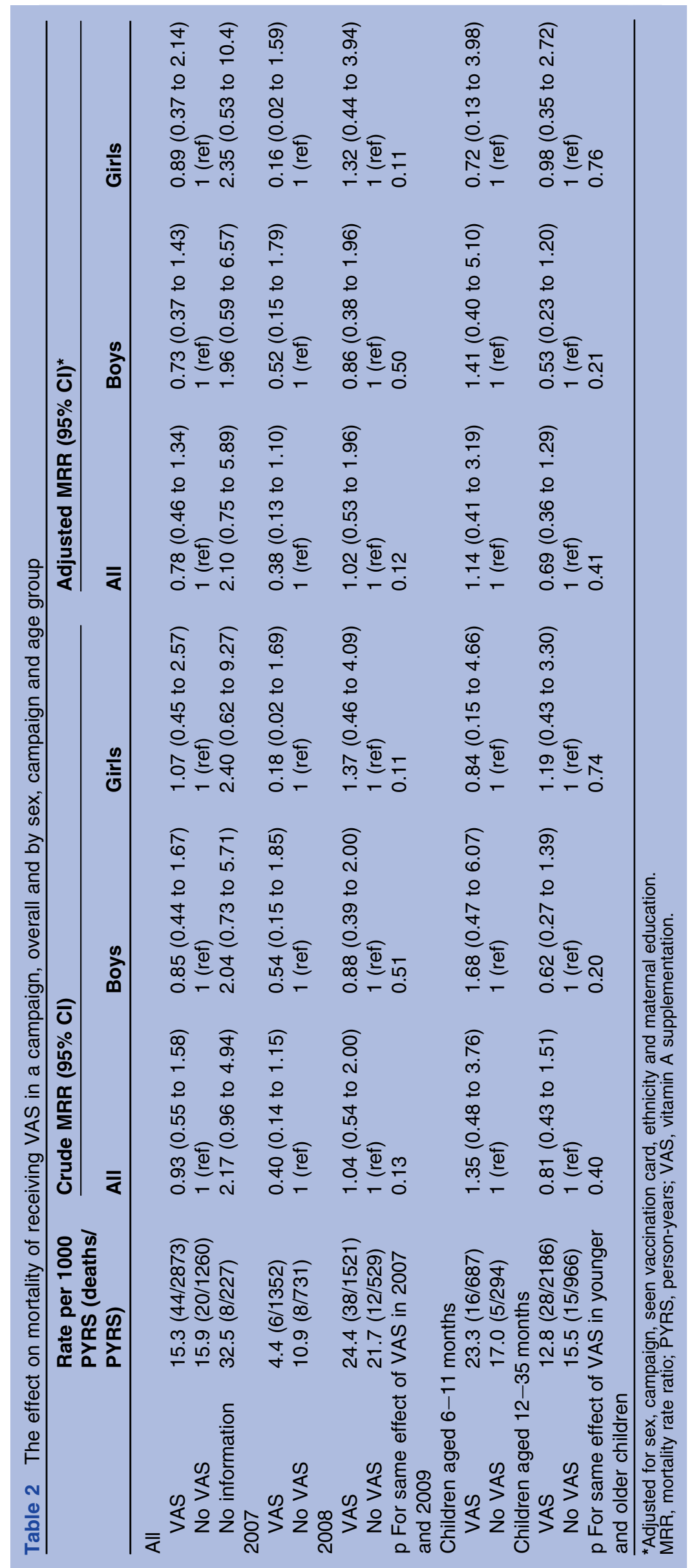


adjusted MRR was $1.29(0.52 ; 3.22)(\mathrm{p}=0.04$ for different effect in MV and DTP recipients). The pattern was similar in boys and girls (table 3). The small group of non-supplemented children with OPV as the most recent vaccine had lower mortality than all other groups (table 3).

\section{Effect of supplementation in the 2007 campaign on survival} after the 2008 campaign

Supplementation in the 2007 campaign was associated with improved survival after the 2008 campaign. Among 3520 children supplemented in 2008, 69\% (2441) had been eligible for supplementation in 2007; 1435 (59\%) had received VAS, 917 (38\%) had not and $89(4 \%)$ had no information on participation. After the 2008 campaign, children supplemented in both campaigns had lower mortality (8 deaths/623 person-years) than children who had not received VAS in 2007 but received VAS in 2008 (15 deaths/394 person-years), the adjusted MRR being $0.34(0.14 ; 0.80)$. This beneficial effect of prior VAS was more pronounced for girls $(\mathrm{MRR}=0.14$ $(0.03 ; 0.68))$ than for boys $(\mathrm{MRR}=0.59(0.20 ; 1.77))$ (test of interaction between sex and VAS, $\mathrm{p}=0.14$ ).

\section{Cause of death}

We aimed to examine the effect of VAS on all-cause mortality. However, from May 2008 to January 2009 a cholera epidemic occurred. ${ }^{22}$ This epidemic could potentially explain the higher mortality after the 2008 campaign. Verbal autopsies were conducted for 66 of the 72 deaths, for the remaining six the family had moved prior to the interview. One death which occurred after receiving VAS in 2008 was classified as due to cholera. Further 10 deaths $(15 \%)$ were due to diarrhoeal disease, one after the 2007 campaign (no VAS) and nine after the 2008 campaign (eight VAS and one no VAS). The peak of the epidemic occurred in September 2008 ${ }^{22}$; five diarrhoeal deaths in 2008 occurred in August and September (all VAS) and four deaths (three VAS and one no VAS) occurred in the late epidemic in November and December where few cholera cases were detected. Campaign participation had no significant effect on the risk of death due to diarrhoea, the MRR being 1.87 $(0.40 ; 8.71)$. The other causes of death were prolonged disease with failure to thrive and anaemia, 18 (27\%, 14 VAS, three no VAS and one no information); respiratory infection, 17 (26\%, nine VAS, six no VAS and two no information); malaria, 14 (21\%, five VAS, seven no VAS and two no information); fever of unknown origin, three (5\%, two VAS and one no VAS); cerebral palsy, two (3\%, one VAS and one no VAS) and accident, one (2\%, VAS). There were significantly more malaria deaths among the non-supplemented and the children with no information, the MRRs being $3.26(1.03 ; 10.3)$ and 5.43 (1.05; 28.2), respectively, presumably reflecting that they were less likely to seek early treatment or to be absent in the rural areas where the incidence of severe and untreated malaria would be higher.

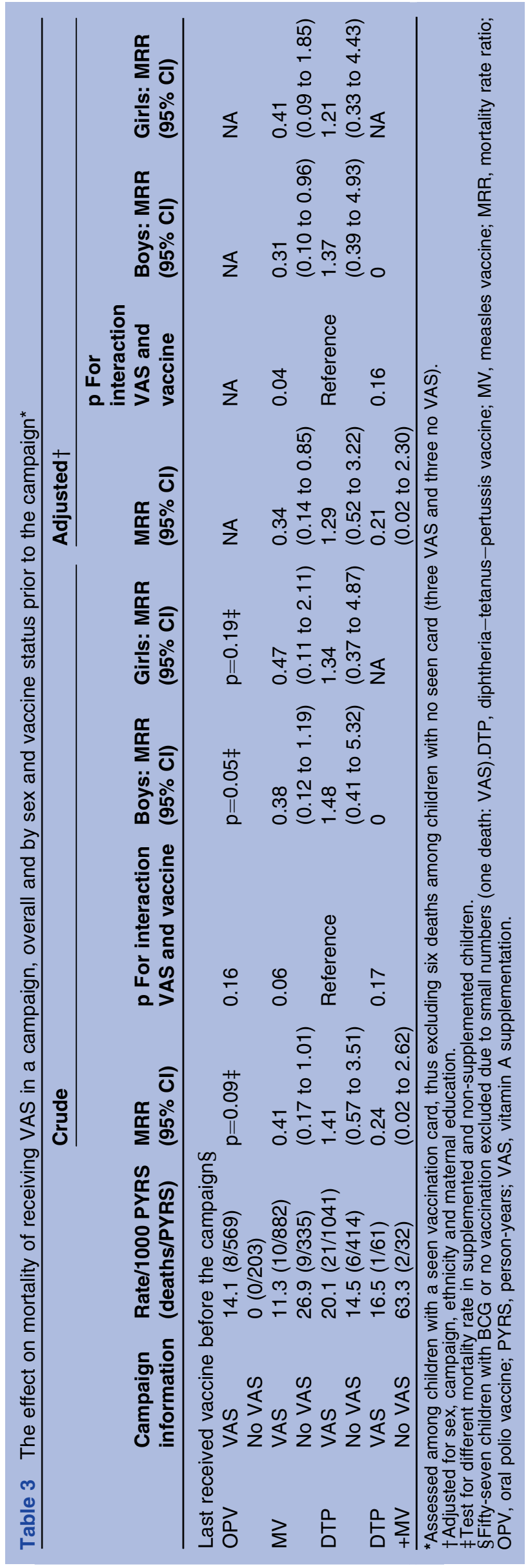




\section{DISCUSSION}

Based on the marked mortality reductions seen in trial in the 1980s and 1990s, VAS has become a high-priority intervention, expected to reduce overall child mortality. However, according to the preliminary report, a recent trial VAS in 1 million Indian children has not shown the same beneficial effect. ${ }^{2}$

In our study, children receiving VAS in a campaign had $7 \%(-58 \% ; 45 \%)$ lower mortality compared with nonsupplemented children in the crude analysis and 22\% $(-34 \% ; 54 \%)$ lower mortality in the adjusted analysis. However, this is unlikely to represent the true effect of VAS since there are many reasons that non-supplemented children may have had higher mortality, for example, selection bias, travelling to the rural areas, less access to care and a higher risk of dying of malaria. More important, our observational study identified differential effects of VAS by season and vaccination status; for example, supplemented children did not have lower mortality than non-supplemented children in the rainy season and among children who had DTP as the most recent vaccination.

\section{Strengths and weaknesses}

The strengths of our study include that information was collected on the individual level and that the children were followed prospectively. By only allowing the children to contribute observation time in the VAS or no VAS group from the day the information was obtained, we avoided introducing survival bias in the analysis (ie, obtaining positive information only from surviving children). ${ }^{23}$

Vitamin A content was assessed in the expired capsules which some campaign recipients received in 2007. Though the content was lower than the original $200000 \mathrm{IU}$, the content was still high. Hence, we believe that all supplemented children received a relevant dose of vitamin A.

Mortality was higher in children for whom we do not have information on the campaign participation and also a substantial number of children died prior to us getting the information on their participation in the VAS campaign. The main reason for not getting any information was that the family had travelled to the rural areas, where mortality is higher. ${ }^{24}$ According to national data, VAS coverage was considerably higher outside the capital and hence outside the urban study area (personal communication, Sidu Biai, WHO, Bissau). Hence, the children for whom we did not obtain information may have been supplemented in the rural areas and if anything more of the deaths among travelling children may have occurred in supplemented than non-supplemented children.

\section{Consistency with other studies}

Apart from our previous studies from Bissau, ${ }^{11} 1924$ there has been no evaluation of the impact of VAS campaigns on mortality based on individual level data. We have previously shown that the effect of VAS was more beneficial when provided with MV than with DTP vaccine. ${ }^{11}$ We have also gathered evidence that the effect of VAS versus placebo was more beneficial in girls with MV than in girls with DTP as their most recent vaccine. ${ }^{8}$ In the present study, we compared campaign participants with non-participants rather than VAS with placebo recipients, which may have introduced selection biases in the comparison of VAS recipients and nonrecipients. However, the varying effect by vaccination status is unlikely to be explained by simple selection bias. We had expected that a negative effect of VAS with DTP would be most pronounced for girls. ${ }^{825} 26$ This was not the case. However, recent studies have indicated that mortality after VAS is lowered in girls who have received VAS on a previous occasion, ${ }^{20} 21$ and this was also the case in the present study. Thus, repeated dosing may have alleviated the negative effects of VAS with DTP in girls.

Mortality is usually $15 \%$ higher in the rainy (May to November) than in the dry season, ${ }^{27}$ and though this may explain some of the differences in mortality after the 2007 and 2008 campaigns, it is unlikely to explain the threefold higher mortality. The difference may partly be due to a more beneficial effect of VAS in the dry than in the rainy season (table 2). We have previously found strong differences in the effect of neonatal VAS by season. In a randomised trial, neonatal VAS benefited infant survival among normal birth weight neonates in the dry season but was associated with increased mortality in the rainy season. ${ }^{18}$ However, we found no seasonal differences in the effect of neonatal VAS to low birth weight infants ${ }^{26}$ and no strong seasonal pattern was seen in Ghana where an analysis by season has been reported. ${ }^{28}$ Though numbers were small and the difference was not statistically significant, VAS appeared to be associated with an increased risk of dying of diarrhoea during the cholera epidemic in 2008. This contrasts with a recent meta-analysis that estimates $28 \% \quad(9 \% ; 43 \%)$ reduction in diarrhoea-related mortality. ${ }^{1}$

The effect of VAS on mortality has most often been ascribed to prevention and treatment of vitamin A deficiency. $^{29}$ We have previously found poorer vitamin A status in children of non-educated mothers. ${ }^{13}$ In the current study, we found no evidence of a beneficial effect limited to lower socioeconomic groups, which presumably are the most vitamin A deficient.

\section{Interpretation}

The present study as well as previous observations support that the effect of VAS differs depending on vaccination status being beneficial when provided after $\mathrm{MV}^{3}{ }^{8}$ but not after DTP. This is unlikely to be explained by selection bias since bias is unlikely to work in different direction for children who had MV or DTP as their most recent vaccine. It is also unlikely to be explained by differences in vitamin A deficiency.

We do not know what may have caused the observed tendency for a differential effect of VAS on mortality 
between the two campaigns. Previous studies have demonstrated seasonal variance in pathogen prevalences with distinct season-related epidemics of rotavirus, ${ }^{30}$ cryptosporidium, ${ }^{31}$ respiratory syncytial virus ${ }^{32}$ as well as a malaria. ${ }^{33}$ The effect of VAS has been show to differ depending on the pathogen, ${ }^{34}$ and varying pathogen prevalences may therefore contribute to the seasonal differences. Based on the verbal autopsy data, VAS may have been associated with a higher risk of dying from cholera/diarrhoea during the cholera epidemic in 2008. An alternative explanation is the generally increased prevalence of infectious diseases in the rainy season, which may lead to impaired uptake and increased excretion of vitamin A. ${ }^{35}$ This could explain the lack of effect during the rainy season but would not explain the vaccine differential effect. Regardless of the mechanism, the potential differential seasonal effects of VAS deserve further investigation.

\section{Implications and conclusions}

Our present study highlights the importance of continuing to evaluate the effect of presumed beneficial interventions. The circumstances under which the intervention is being implemented may differ from the circumstances under which it was originally tested. The present study as well as several previous studies suggest that the effect of VAS is beneficial when administered with or after $\mathrm{MV}^{3} 81136$ but not when administered with or after DTP/pentavalent. ${ }^{4} 11$ 37-41 Hence, in a situation where children with DTP as the most recent vaccination predominate, the overall effect of VAS campaigns could be negative. From this perspective, it may be no coincidence that the recent huge trial of 1 million children in India, where a booster dose of DTP is recommended at 18 months of age, ${ }^{42}$ showed no beneficial effect of biannual VAS. ${ }^{2}$

Our research questions the current focus on up-scaling VAS and integrating interventions to achieve higher coverage. ${ }^{43}$ We first need to understand under which conditions VAS does not have a beneficial effect. In a recent meta-analysis, ${ }^{144}$ it is argued that no further placebo-controlled trials are needed. The meta-analysis is essentially based on trials from the late 1980s and early 1990s and to declare that no further trials are needed presupposes that it can be shown that the effect has not changed over time. The only recent trials are the megatrial from India showing no effect ${ }^{2}$ and a placebocontrolled trial of VAS administered with vaccines in Guinea-Bissau also showing no overall but sex-differential effects (unpublished data). Hence, the effect of VAS may have changed, and placebo-controlled trials may be needed to clarify whether season matters and with which vaccines VAS should be given.

Funding This work was supported by DANIDA (through grant 104.Dan.8-920), the Danish Medical Research Council, Graduate School of International Health, the Aase and Ejnar Danielsens Foundation, Danish Medical Associations Research foundation and Else and Mogens Wedell-Wedellsborgs Foundation. The Bandim Health Project received support from DANIDA and the Danish National Research Foundation. PA holds a research professorship grant from the Novo Nordisk Foundation. The sponsors had no role in the study design, data collection, data analysis, data interpretation or the writing of the report.

Competing interests None.

Contributors ABF, PA and CSB planned the study. ABF, CB, IB, SB-S supervised the campaign registration, data entry and follow-up. $C M$ was responsible for trial $B$ and $J A$ was responsible for trials $A$ and $C$ and both contributed the vaccination data. ABF was responsible for the statistical analysis with help from BMB. ABF wrote the first draft of the paper and has the primary responsibility for the final content. All authors contributed to and approved the final manuscript.

Provenance and peer review Not commissioned; externally peer reviewed.

Data sharing statement No additional data available.

\section{REFERENCES}

1. Mayo-Wilson E, Imdad A, Herzer K, et al. Vitamin A supplements for preventing mortality, illness, and blindness in children aged under 5 : systematic review and meta-analysis. BMJ 2011;343:d5094.

2. Awasthi S, Peto R, Read S, et al. Six-monthly augmented vitamin A supplementation from 1 to 6 years of age: block randomized trial in one million children in northern India. Presented at the 2007 micronutrient forum. http://www.micronutrientforum.org/Meeting2007/ Tuesday/T1445\%20Awasthi.pdf (accessed 24 Nov 2011).

3. Benn CS, Bale C, Sommerfelt $\mathrm{H}$, et al. Hypothesis: vitamin A supplementation and childhood mortality: amplification of the non-specific effects of vaccines? Int J Epidemiol 2003;32:822-8.

4. Anon. Randomised trial to assess benefits and safety of vitamin A supplementation linked to immunisation in early infancy. WHO/CHD immunisation-linked vitamin A supplementation study group. Lancet 1998;352:1257-63.

5. West J, Katz J, Shrestha SR, et al. Mortality of infants $<6$ mo of age supplemented with vitamin A: a randomized, double-masked trial in Nepal. Am J Clin Nutr 1995;62:143-8.

6. UNICEF and World Health Organization. Immunisation Summary; A Statistical Reference Containing Data Through. 2009. http://www. childinfo.org/files/32775_UNICEF.pdf (accessed 24 Nov 2011).

7. Anon. Vitamin A supplementation in northern Ghana: effects on clinic attendances, hospital admissions, and child mortality. Ghana VAST Study Team. Lancet 1993;342:7-12.

8. Benn CS, Aaby P, Nielsen J, et al. Does vitamin A supplementation interact with routine vaccinations? An analysis of the Ghana vitamin A supplementation trial. Am J Clin Nutr 2009;90:629-39.

9. World Health Organization. Guideline: vitamin A supplementation in infants and children 6-59 months of age. http://www.who.int/nutrition/ publications/micronutrients/guidelines/vas_6to59_months/en/index. html (accessed 24 Nov 2011).

10. World Health Organization. Integration of vitamin A supplementation with immunization. Wkly Epidemiol Rec 1999;74:1-6.

11. Benn CS, Martins C, Rodrigues A, et al. The effect of vitamin A supplementation administered with missing vaccines during national immunization days in Guinea-Bissau. Int $J$ Epidemiol 2009;38:304-11.

12. World Health Organization. Global Prevalence of Vitamin A Deficiency in Populations at Risk 1995-2005: WHO Global Database on Vitamin A Deficiency. http://whqlibdoc.who.int/ publications/2009/9789241598019_eng.pdf (accessed 24 Nov 2011).

13. Fisker $A B$, Lisse I, Aaby $P$, et al. Impact of neonatal vitamin A supplementation with BCG vaccine on vitamin A status at 6 weeks and 4 months of age. Am J Clin Nutr 2007;86:1032-9.

14. Aaby P, Martins CL, Garly ML, et al. Non-specific effects of standard measles vaccine at 4.5 and 9 months of age on childhood mortality: randomised controlled trial. BMJ 2010;341:c6495.

15. Agergaard J, Nante E, Poulstrup G, et al. Diphtheria-tetanuspertussis vaccine administered simultaneously with measles vaccine is associated with increased morbidity and poor growth in girls. A randomised trial from Guinea-Bissau. Vaccine 2011;29:487-500.

16. Aaby $P$, Jensen $H$, Rodrigues $A$, et al. Divergent female-male mortality ratios associated with different routine vaccinations among female-male twin pairs. Int J Epidemiol 2004;33:367-73.

17. INDEPTH Network. INDEPTH Standardized Verbal Autopsy Questionnaire. http://www.indepth-network.org/index.php? option $=$ com content\&task $=$ view\&id $=96 \&$ Itemid $=184$ (accessed 24 Nov 2011).

18. Benn CS, Diness BR, Roth A, et al. Effect of 50,000 IU vitamin A given with BCG vaccine on mortality in infants in Guinea-Bissau: randomised placebo controlled trial. BMJ 2008;336:1416-20.

19. Benn CS, Martins C, Rodrigues A, et al. Randomised study of effect of different doses of vitamin A on childhood morbidity and mortality. BMJ 2005;331:1428-32. 
20. Fisker AB, Aaby P, Rodrigues A, et al. Vitamin A supplementation at birth might prime the response to subsequent vitamin A supplements in girls. Three year follow-up of a randomized trial. PLOS ONE 2011;6: e23265.

21. Yakymenko D, Benn CS, Martins C, et al. The impact of different doses of vitamin A supplementation on male and female mortality. A randomised trial from Guinea-Bissau. BMC Pediatr 2011;11:77.

22. Luquero FJ, Banga CN, Remartinez D, et al. Cholera epidemic in Guinea-Bissau (2008): the importance of "place". PLoS ONE 2011;6: e19005.

23. Jensen H, Benn CS, Lisse IM, et al. Survival bias in observational studies of the impact of routine immunizations on childhood survival. Trop Med Int Health 2007;12:5-14.

24. Nielsen J, Benn CS, Bale C, et al. Vitamin A supplementation during waremergency in Guinea-Bissau 1998-1999. Acta Trop 2005;93:275-82.

25. Benn CS, Rodrigues A, Yazdanbakhsh M, et al. The effect of highdose vitamin A supplementation administered with BCG vaccine at birth may be modified by subsequent DTP vaccination. Vaccine 2009;27:2891-8.

26. Benn CS, Fisker AB, Napirna BM, et al. Vitamin A supplementation and $B C G$ vaccination at birth in low birthweight neonates: two by two factorial randomised controlled trial. BMJ 2010;340:c1101.

27. Veirum JE, Biai S, Jakobsen M, et al. Persisting high hospital and community childhood mortality in an urban setting in Guinea-Bissau. Acta Paediatr 2007;96:1526-30.

28. Ross DA, Kirkwood BR, Binka FN, et al. Child morbidity and mortality following vitamin A supplementation in Ghana: time since dosing, number of doses, and time of year. Am J Public Health 1995;85:1246-51.

29. Humphrey JH, West KP Jr, Sommer A. Vitamin A deficiency and attributable mortality among under-5-year-olds. Bull World Health Organ 1992;70:225-32.

30. Fischer TK, Aaby P, Molbak K, et al. Rotavirus disease in GuineaBissau, West Africa: a review of longitudinal community and hospital studies. J Infect Dis 2010;202(Suppl):S239-42.

31. Perch M, Sodemann M, Jakobsen MS, et al. Seven years' experience with cryptosporidium parvum in Guinea-Bissau, West Africa. Ann Trop Paediatr 2001;21:313-18.

32. Stensballe LG, Nante $\mathrm{E}$, Jensen IP, et al. Acute lower respiratory tract infections and respiratory syncytial virus in infants in Guinea-Bissau: a beneficial effect of BCG vaccination for girls community based case-control study. Vaccine 2005;23:1251-7.

33. Rodrigues A, Schellenberg JA, Kofoed PE, et al. Changing pattern of malaria in Bissau, Guinea Bissau. Trop Med Int Health 2008;13:410-17.

34. Long KZ, Estrada-Garcia T, Rosado JL, et al. The effect of vitamin A supplementation on the intestinal immune response in Mexican children is modified by pathogen infections and diarrhea. $J$ Nutr 2006; 136:1365-70.

35. Alvarez JO, Salazar-Lindo E, Kohatsu J, et al. Urinary excretion of retinol in children with acute diarrhea. Am J Clin Nutr 1995:61:1273-6.

36. Benn CS, Aaby P, Bale C, et al. Randomised trial of effect of vitamin A supplementation on antibody response to measles vaccine in Guinea-Bissau, West Africa. Lancet 1997;350:101-5.

37. Newton S, Cousens S, Owusu-Agyei S, et al. Vitamin a supplementation does not affect infants' immune responses to polio and tetanus vaccines. $J$ Nutr 2005;135:2669-73.

38. Newton S, Owusu-Agyei S, Filteau S, et al. Vitamin A supplements are well tolerated with the pentavalent vaccine. Vaccine 2008;26:6608-13.

39. Darboe MK, Thurnham DI, Morgan G, et al. Effectiveness of an early supplementation scheme of high-dose vitamin A versus standard WHO protocol in Gambian mothers and infants: a randomised controlled trial. Lancet 2007;369:2088-96.

40. Mahalanabis D, Rahman MM, Wahed MA, et al. Vitamin A megadoses during early infancy on serum retinol concentration and acute side effects and residual effects on 6 month follow-up. Nutr Res 1997:17:649-59.

41. Rahman MM, Mahalanabis D, Wahed MA, et al. Administration of 25,000 IU vitamin A doses at routine immunisation in young infants. Eur J Clin Nutr 1995;49:439-45.

42. World Health Organisation. Vaccination Schedule In India. http:// whoindia.org/LinkFiles/Routine Immunization National Immunization_Schedule.pdf (accessed 24 Nov 2011).

43. UNICEF. Vitamin A supplementation. A decade of progress. http:// www.unicef.org/publications/index_39363.html (accessed 24 Nov 2011).

44. Thorne-Lyman A, Fawzi WW. Improving child survival through vitamin A supplementation. BMJ 2011;343:d5294. 